\title{
Fangorn Forest (F2): a machine learning approach to classify genes and genera in the family Geminiviridae
}

\author{
José Cleydson F. Silva ${ }^{1,3}$, Thales F. M. Carvalho', Elizabeth P. B. Fontes ${ }^{2,3^{*}}$ and Fabio R. Cerqueira ${ }^{1,4^{*}}$
}

\begin{abstract}
Background: Geminiviruses infect a broad range of cultivated and non-cultivated plants, causing significant economic losses worldwide. The studies of the diversity of species, taxonomy, mechanisms of evolution, geographic distribution, and mechanisms of interaction of these pathogens with the host have greatly increased in recent years. Furthermore, the use of rolling circle amplification (RCA) and advanced metagenomics approaches have enabled the elucidation of viromes and the identification of many viral agents in a large number of plant species. As a result, determining the nomenclature and taxonomically classifying geminiviruses turned into complex tasks. In addition, the gene responsible for viral replication (particularly, the viruses belonging to the genus Mastrevirus) may be spliced due to the use of the transcriptional/splicing machinery in the host cells. However, the current tools have limitations concerning the identification of introns.

Results: This study proposes a new method, designated Fangorn Forest (F2), based on machine learning approaches to classify genera using an ab initio approach, i.e., using only the genomic sequence, as well as to predict and classify genes in the family Geminiviridae. In this investigation, nine genera of the family Geminiviridae and their related satellite DNAs were selected. We obtained two training sets, one for genus classification, containing attributes extracted from the complete genome of geminiviruses, while the other was made up to classify geminivirus genes, containing attributes extracted from ORFs taken from the complete genomes cited above. Three ML algorithms were applied on those datasets to build the predictive models: support vector machines, using the sequential minimal optimization training approach, random forest (RF), and multilayer perceptron. RF demonstrated a very high predictive power, achieving $0.966,0.964$, and 0.995 of precision, recall, and area under the curve (AUC), respectively, for genus classification. For gene classification, RF could reach 0.983, 0.983, and 0.998 of precision, recall, and AUC, respectively.
\end{abstract}

Conclusions: Therefore, Fangorn Forest is proven to be an efficient method for classifying genera of the family Geminiviridae with high precision and effective gene prediction and classification. The method is freely accessible at www.geminivirus.org:8080/geminivirusdw/discoveryGeminivirus.jsp.

Keywords: Geminivirus; machine learning, Gene classification, Genus classification, Random Forest, Multilayer perceptron, Support vector machines

\footnotetext{
*Correspondence: bbfontes@ufv.br; frcerqueira@id.uff.br

${ }^{2}$ National Institute of Science and Technology in Plant-Pest Interactions/

BIOAGRO, Campus Universitário, Viçosa, Minas Gerais 36570-900, Brazil

'Department of Informatics, Universidade Federal de Viçosa, Viçosa, Minas

Gerais 36570-900, Brazil

Full list of author information is available at the end of the article
} 


\section{Background}

Geminiviridae is one of the largest and most successfully plant virus families. This family comprises viruses with single-strand DNA genome encapsulated in twinned icosahedral particles. Geminiviruses infect several species of cultivated and ornamental plants as well as weeds, causing significant economic losses in agriculture and food safety worldwide [1]. The family Geminiviridae comprises nine genera: Begomovirus, Mastrevirus, Becurtovirus, Curtovirus, Turncurtovirus, Eragrovirus, Topocuvirus, Capulavirus, and Graglovirus [2-4]. Geminivirus genomes are comprised of a genomic component called DNA-A. Viruses of the Begomovirus genus are exceptions. Their genomes can present only the component DNA-A (monopartite), similarly to other geminiviruses, or two components: DNA-Aand DNA-B (bipartite). The component DNA-A may be transmitted by the silverleaf whitefly (Bemisia tabaci of biotypes A or B), particularly for begomoviruses; by leafhoppers (mastreviruses, becurtoviruses, and curtoviruses), and by treehoppers (topocuviruses) $[1,2,5,6]$. The genera Eragrovirus and Turncurtovirus have no known vector yet. The genomes of bipartite Begomovirus are mostly found in the New World, while monopartite ones (made up of only DNA-A) are commonly found in the Old World [7-9].

Recent studies report the first occurrence of monopartite geminivirus (begomoviruses) infecting tomatoes in Peru and Ecuador [10]. Conversely, bipartite begomoviruses have been identified in the Old World (Madagascar) infecting Asystasia gangetica and associated with mosaic disease in Coccinia grandis in India [11-13]. Overall, diseases caused by geminiviruses have had economic and social impacts in several continents. For example, in Europe, tomato plants have been infected by the tomato yellow leaf curl virus disease (TYLCD) and wheat has been severely inflicted by the wheat dwarf virus disease (WDVD) [14-16]. In Africa, the cassava mosaic disease (CMD) and the maize streak disease (MSD) have been reported $[17,18]$. There have also been occurrences of the cotton leaf curl disease (CLCuD) and the chickpea chlorotic dwarf disease in Asia, as well as the bean golden mosaic disease (BGMD) in the Americas [19-21].

The genomic organization of geminiviruses is highly conserved. However, the species are genetically divergent, encoding two to seven genes, with long and short intergenic regions and a common region between DNA-A and DNA-B [2]. DNA-A encodes CP (capsid proteins), Rep (a protein associated with replication), TrAP (transcriptional activator protein and gene silencing suppressor), REn (replication enhancer protein), Reg (gene regulator), Sd (or AC4, symptom determinant and gene silencing suppressor), and AC5 (recently studied and functionally described as a determinant of pathogenicity that suppresses antiviral defenses based on RNA silencing) [2, 22]. Furthermore, monopartite geminiviruses in the Old World contain a pre-coat protein (V2) related to movement and transport of viral genome in the plant.

DNA-B (reported for begomovirus) is responsible for the transport and movement of viral DNA in the plant and codes two proteins, MP (movement protein) and NSP (nuclear transport protein). NSP facilitates the intracellular transport of viral DNA from the nucleus to the cytoplasm and acts in concert with MP to move the viral DNA to the adjacent, uninfected cells [23]. In some cases, geminiviruses may be associated with beta satellite (DNA-Beta) or alpha satellite DNA (DNA-Alpha) [24]. Beta satellites are DNA molecules with approximately $1.35 \mathrm{~kb}$, and code a single ORF betaC1 (pathogenicity determinant protein), which acts in the development of symptoms, modulation of virus host range, and host defense response [25-27]. In contrast, alpha satellites are capable of autonomous replication but are dependent on geminiviruses for systemic infection and vector transmission [28, 29]. The genome of alpha satellites contains approximately $1.37 \mathrm{~kb}$ and codes a single Rep protein.

Recent researches have shown the high diversity of geminivirus species, multiple hosts, and geographic distribution in various regions of the Old and New Worlds [2, 30-32]. Currently, high-throughput sequencing methods, advanced metagenomics approaches, and different bioinformatics tools have enabled elucidating viromes and identifying many viral agents in a large number of plant species. In addition, using the rolling circle amplification (RCA) approach [33], thousands of sequences or complete genomes have been amplified, sequenced, and made available in public databases (GenBank NCBI, geminivirus.org). Currently, geminiviruses are classified based on the type of insect vector, host range, phylogenetic reconstruction, and genomic organization [2]. Therefore, geminivirus classification requires knowledge of taxonomy and bioinformatics since different computational tools and algorithms can be used. For example, the algorithms Muscle, MAFFT, ClustalW, and BLAST are often used for alignment of sequences [34-37]. Methods, including neighbor-joining, maximum parsimony, maximum likelihood, and Bayesian inference, are also used to obtain phylogenetic reconstruction $[3,4,38]$. Other approaches using pairwise sequence comparisons are also widely employed. Those comparisons are used by the software SDT [39] and analyzed according to the taxonomic criterion of each genus. Several previous works have applied those computational tools to provide taxonomic reviews [2-4, 30-32, 40]. Guidelines and protocols have been proposed to demarcate and classify species for Becurtovirus, Eragrovirus, and Turncurtovirus [2]. Similarly, criteria have also been proposed for begomoviruses and mastreviruses [30, 31]. In order to evaluate the genomic organization, the Open Reading 
Frames (ORFs) and their respective positions in the genome must be first obtained. In this step, ORFs are predicted by the ORF finder tool (https:// www.ncbi.nlm.nih.gov/orffinder/), which, although widely used, has limitations in identifying introns of this family. Other consolidated tools, such as AUGUSTUS (http:// augustus.gobics.de/), Geneid (http://genome.crg.es/software/geneid/index.html) and Prodigal (https://github.com/hyattpd/Prodigal), are still limited to identify all ORFs that are encoded by the geminivirus genomes. Even though the computer programs cited above are robust and help taxonomic classification, they are of general purpose, i.e., they were not designed taking the peculiarities of geminivirus genomes into account. Furthermore, they often use databases with non-standardized, non-curated sequences with frequent annotation errors. Still, in general, the required methods are not integrated. Such integration would facilitate automating the data analysis process and decision-making.

We hereby present an in silico prediction approach, called Fangorn Forest (F2), capable of classifying genera and genes in the Geminiviridae family based on machine learning (ML) methods. F2 uses only genomic characteristics common to any viral genome to build classification models. In this research, all genera (nine) of the family Geminiviridae and their related satellite DNAs were considered. The proposed method is proven to be highly accurate, as the machine learning models used yielded very high values of precision, recall, and area under the ROC curve (AUC) for the classification tasks. F2 integrates the set of computational tools of the data warehouse www.geminivir us.org:8080/geminivirusdw/discoveryGeminivirus.jsp [41] .

\section{Methods}

\section{Data source}

Initially, genome sequences of plant viruses were retrieved from the GenBank database for composing the negative class (non-geminiviruses) of the training set for family classification.The non-geminivirus class is composed by DNA sequence of different families of plant viruses. This class consists of double-stranded DNA sequences (Caulimovidae), double-stranded RNA viruses (Amalgaviridae, Fijiviridae, Oryzaviridae), single-stranded DNA (Nanoviridae), negative sense single-stranded RNA viruses (Ophioviridae) and positive sense single-chain RNA viruses (Benyviridae, Bromoviridae, Closteroviridae, Luteoviridae, Potyviridae, Tombusviridae, Virgaviridae) (http://viralzone.expasy.org/). This class was intended to distinguish genomic sequences of geminiviruses from other plant viruses.

Complete genome sequences of species from eight genera in the Geminivividae family as well as satellite DNAs were used to create the positive class of the training set instances for Geminiviridae family classification (mentioned before) and genus classification. All sequences were obtained from the geminivirus.org curated repository [40]. The sequences of Begomovirus, Mastrevirus, Becurtovirus, Curtovirus, Turncurtovirus, Eragrovirus, Capulavirus, and Graglovirus were defined according to taxonomic reviews $[2-4,30-32,41,42]$. Additionally, the complete genomes of betasatellites were chosen in conformity with the study of Briddon et al. [31], while sequences of alphasatellites and DNA-B were randomly selected from the geminivirus.org repository. The genus Topocuvirus was not selected because has only one sequence deposited in GenBank database.

A family test set was also created using sequences of GenBank database. These sequences. Which were not present in the training set, were used only for the negative class. The sequences used in the positive class were retrieved from geminivirus.org. Also a genus test set was also created using sequences of geminivirus.org, which were not present in the training set. Therefore, four datasets were created. Two datasets (for training and test) comprised of instances of two classes (geminiviruses and non-geminiviruses) and two resultant datasets (for training and test) were comprised of instances of ten classes: begomoviruses/DNA-B, mastreviruses, becurtoviruses, curtoviruses, turncurtoviruses, eragroviruses, capulaviruses, grabloviruses, alphasatellites, and betasatellites.

After creating datasets related to genus classification, we also built training and test sets for gene (ORF) classification. To make up the ORF training set, we selected ORFs contained in the genomes and used in the aforementioned genus training set. In the same way, the ORF test set was composed of ORFs extracted from the same sequences considered to build the genus test set mentioned above. The instance classes of the resultant datasets related to ORF classification are: betaC1, alphaRep, Rep, TrAP, REn, Sd/p.sd, AC5, CP, pre-coat, Reg, MP, and NSP.

As could be noted, we perform a multi-class classification in both genus and ORF classification. Figure 1 shows a phylogenetic tree built with the genomic sequences used in the training sets. Notice that DNA-A and DNA-B are from the genus Begomovirus, i.e., both DNA-A and DNA$B$ sequences give rise to instances from this genus. The number of instances in each class, composing the training/test sets for family, genus and ORF classification, is shown in Additional file 1: Table S1. Additional file 2 shows the accession numbers of the complete genomes used to create the datasets.

\section{Data quality}

The data available in public databases may contain non-standardized, non-curated sequences, with possible annotation errors, and, consequently, may be inappropriate to build training sets. The sequences used for the 


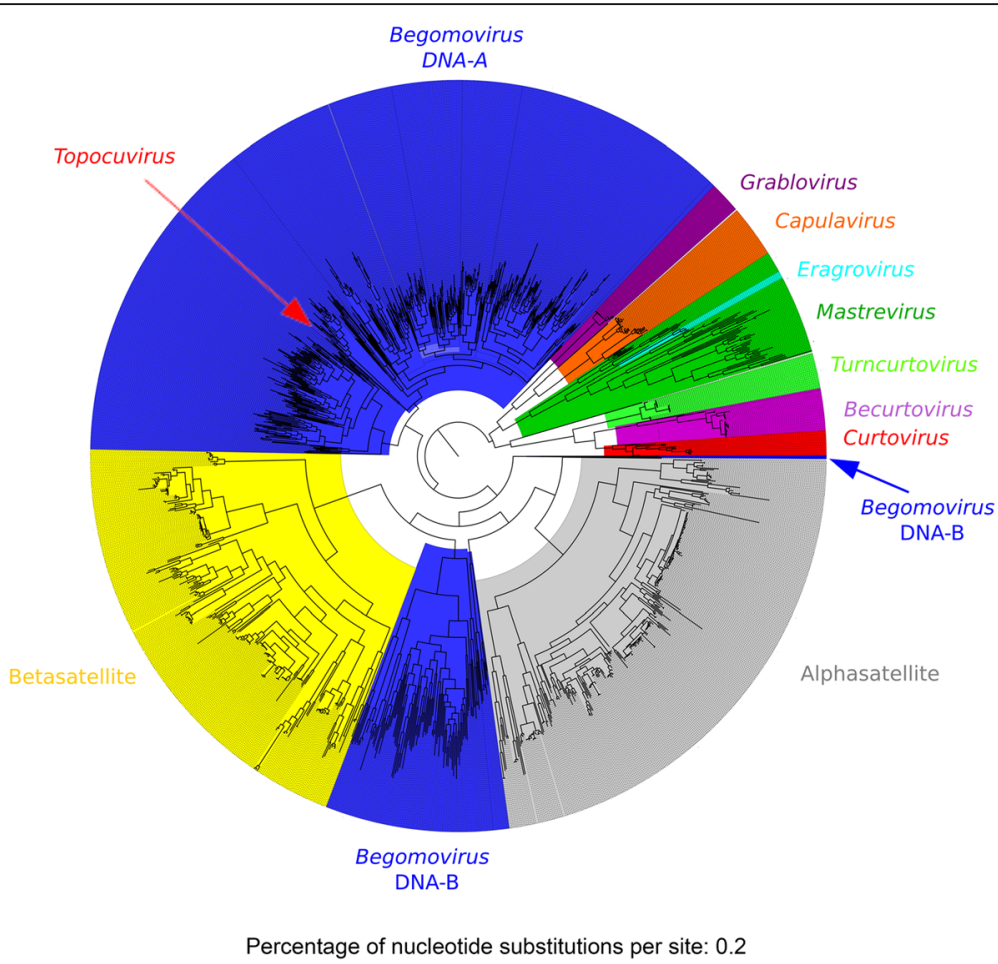

Fig. 1 Phylogenetic reconstruction of the Geminiviridae family and satellite DNAs. To perform the phylogenetic reconstruction of geminiviruses, all genomic sequences belonging to the genus training set were used. Sequences were aligned using the MAFFT algorithm. The phylogenetic reconstruction was obtained through the program FastTree version 2.1.7. The phylogenetic tree was visualized and edited using the program FigTree $\mathbf{1 . 4 . 2}$

training and test sets should fit into the following criteria, which were established and implemented in www.geminivirus.org:

(i) The genomic sequences must start with the conserved 5' end nucleotides (AC) of the Rep cleavage site;

(ii) the last seven nucleotides have to be the conserved sequence TAATATT that corresponds to the initial nucleotides of the replication origin TAATATTAC [43]. Notice that we standardized all genome sequences, which are circular, cutting them between TAATATT and AC;

(iii) the sequence length must be a value within an interval predefined for each genus (Table 1);

(iv) the ORFs must contain a start codon as well as a stop codon, and must not be truncated (no additional stop codon in between);

(v) ORF annotation errors, including wrong acronym as well as start and end positions, are corrected.

In particular, the quality and reliability of the training instances generated from the already-mentioned taxonomic reviews have a high level of confidence, because they are manually curated by a specialized team. Such confidence is fundamental to create good datasets.

\section{Attribute extraction}

The family Geminiviridae comprises plant virus species distributed across nine genera. Interestingly, the genomic organization is highly conserved among those genera. For example, the genes Rep (coded in the virion-complementary strand) and $\mathrm{CP}$ (coded in the virion-sense strand) are common to all genera, and their coordinates in different genomes are approximately equivalent regarding their replication origin [2]. Despite the high conservation of the genomic

Table 1 Minimum and maximum sizes of each genus

\begin{tabular}{lll}
\hline Genus & Minimum size & Maximum size \\
\hline Begomovirus & 2411 & 2959 \\
Mastrevirus & 2425 & 2982 \\
Eragrovirus & 2845 & 2854 \\
Turncurtovirus & 3044 & 3081 \\
Curtovirus & 3011 & 3180 \\
Becurtovirus & 2939 & 2960 \\
Capulavirus & 2550 & 2872 \\
Grablovirus & 3105 & 3205 \\
Unclassified & 2483 & 3308 \\
Betasatellites & 731 & 1552 \\
Alphasatellites & 955 & 1579 \\
\hline
\end{tabular}


structure and particularities of the family Geminiviridae, we selected attributes common to any viral genome so that our considerations could be possibly used in other studies with different species involving the same kind of classification tasks.

The attributes selected to build the family and genus classification models include the proportions of deoxynucleotides. Inspecting the complete genomic sequence, the proportions of adenine (A), thymine $(\mathrm{T})$, cytosine $(\mathrm{C})$, and guanine (G) are calculated. Next, the genomic sequence is split into four equal (or nearly equal) regions (R1, R2, R3, and $\mathrm{R} 4$ ) and, for each one, the proportions of $\mathrm{A}, \mathrm{T}, \mathrm{C}$, and
G as well as the GC content are calculated (Fig. 2a). As a result, we consider 24 attributes for classifying family and, genus, which are presented in Additional file 3: Table S2 and Additional file 4: Table S3, respectively.

To build the gene classification models, the attributes were obtained from each coding DNA sequence (CDS) and its respective amino acid sequence. First, attributes such as ORF orientation in the genome (forward/complement), CDS length, and proportion of nucleotides of the CDS in relation to the complete genome (CDS length/genome length) are extracted. Also, the A, T, C, and $\mathrm{G}$ proportions of the $\mathrm{CDS}$ itself are calculated.

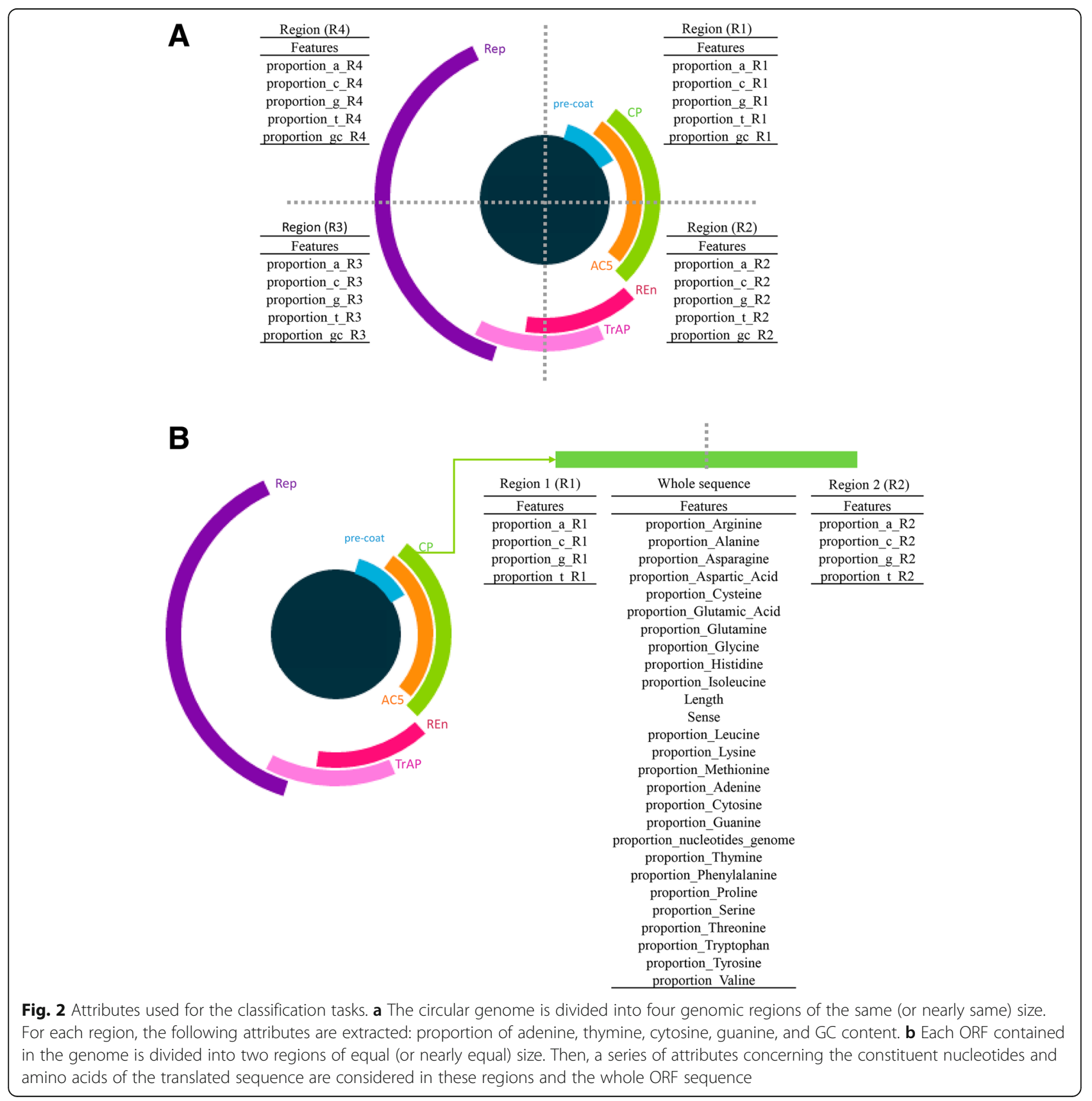


Moreover, the CDS is split into two equal (or nearly equal) regions and, for each of these regions, the proportions of $\mathrm{A}, \mathrm{T}, \mathrm{C}$, and $\mathrm{G}$ are also considered. In addition to those attributes, the proportion of each of the 20 primary amino acids is obtained from the CDS translated sequence (Fig. 2b). Consequently, 35 attributes (see Additional file 5: Table S4) are taken into account.

\section{Attribute evaluation}

Evaluating the attributes extracted from genomic sequences enables identifying which ones help differentiate one genus from another in the classification process. In the same way, measuring the relevance of ORF attributes enables verifying how such attributes contribute to the classification of genes.

Thus, in order to evaluate the importance of each attribute in the training sets, two ranking methods were used: information gain (IG) and RELIEFF [44, 45]. The IG method is based on the shannon entropy and is largely used in many bioinformatics studies [46, 47]. This method assesses the attributes by measuring the information gain they provide in relation to the class attribute. The IG method is defined by IG(Attribute) $=$ Entropy (Class) Entropy (Class $\mid$ Attribute), where the entropy is given by $-\Sigma$ $p_{i} \log _{2} p_{i}$, and $p_{i}$ is the probability of class $i$.

RELIEFF is an extension of RELIEF [48]. RELIEF was coined for binary classification and builds a weight vector $(W)$ of length $p$ (the number of attributes) to represent the relevance of the attributes. This vector starts with zeros and is updated considering the attribute vector $(X)$ of a random instance as well as the attribute vectors $H$ and $M$, representing the closest instance of the same class (hit) and the closest instance of the other class (miss), respectively, using the following update formula:

$$
w_{i}=w_{i}\left(x_{i}-h_{i}\right)^{2}+\left(x_{i}-m_{I}\right)^{2}
$$

Therefore, differences between $X$ and $H$ contribute to diminish the relevance of the attributes, while differences between $X$ and $M$ contribute to augment the weight of attributes. This process is repeated $m$ times (for $m$ sampled instances), and the final values in $W$ are the average of all iterations (at the end, the values in $W$ are divided by $m$ ). Kononenko proposed RELIEFF to overcome some issues of RELIEF [48]. The main improvements were that the update step is made for all instances, not for a sample; instead of taking only one neighbor of each class, $k$ neighbors of each class are taken into account and their contribution is averaged; the algorithm adapts the calculation of $W$ for multiple classes.

To complement the attribute analysis, descriptive statistics and exploratory data analysis were performed. Boxplots, histograms and density plots were created to visualize the distribution of attribute values in each class (Additional file 6).

\section{Defining candidate ORFs}

To predict genes using ML algorithms, we need first to extract candidate ORFs from the input sequence. To this end, we developed an algorithm based on a greedy approach implemented as part of the F2 method, hereby designated Viaduc de Millau (VM) (Fig. 3). Initially, the algorithm identifies all start codons [ATG $\left(5^{\prime} \rightarrow 3^{\prime}\right)$ and CAT $\left.\left(3^{\prime} \rightarrow 5^{\prime}\right)\right]$ and the reading phase in the sense or anti-sense sequence. In the same way, all stop codons [TAA, TAG, TGA $\left(5^{\prime} \rightarrow 3^{\prime}\right)$ and TTA, CTA, TCA $\left.\left(3^{\prime} \rightarrow 5^{\prime}\right)\right]$ are located. In addition, our procedure determines the coordinates where the start codon and stop codon are located in the genome. Each start codon of the sequence in a given sense is paired with stop codons in the same sense. Next, two steps are performed to check some requirements concerning the consistency of each possible ORF (in $5^{\prime} \rightarrow 3^{\prime}$ or $3^{\prime} \rightarrow 5^{\prime}$ ): (i) whether the sequence is in frame; and (ii) whether the translated amino acid sequence is not truncated, and has size greater or equal to 33 amino acids.

However, genes that code different splicing forms in the $3^{\prime} \rightarrow 5^{\prime}$ orientation of genomic sequences of maize streak virus (MSV) have been reported in the family Geminiviridae [49]. In order to find such genes, an algorithm different from previously proposed procedures was performed. To find these ORFs, basic rules of the biological process of mRNA excision were employed in order to precisely identify splicing regions [50]. In this approach, the start and stop codons may or may not be in the same reading phase in the $3^{\prime} \rightarrow 5^{\prime}$ sense. After obtaining sequences of possible ORFs in $3^{\prime} \rightarrow 5^{\prime}$ containing start and stop codons in equal or different sense, the following steps are applied to check some basic requirements as well as typical characteristics of ORFs with introns in genimiviruses: (i) all stop codons in the $3^{\prime} \rightarrow 5^{\prime}$ sense are inspected to verify whether their positions are greater than the position of the respective start codons; (ii) the existence of excision sites (CT and $\mathrm{AC}$ ) is checked; (iii) each candidate CT excision site is paired with all possible AC sites; (iv) the sizes of the two exons (exon 1: minimum 204 $\mathrm{nt}$ and exon 2: minimum $148 \mathrm{nt}$ ) and the intron (minimum $67 \mathrm{nt}$, maximum $102 \mathrm{nt}$ ) are checked; (v) it is inspected whether the amount of pyrimidines is greater than the amount of purines at $50 \mathrm{pb}$ upstream of the AC excision sites; (vi) the minimum length (1000 nt) of the ORF is verified and whether the sequences are in the correct reading phase; (vii) the reverse complement of the sequence is obtained, the candidate CDS is translated, and it is verified if it is not truncated. The restrictions to exon, intron, and sequence sizes were determined in view of the structure of the 


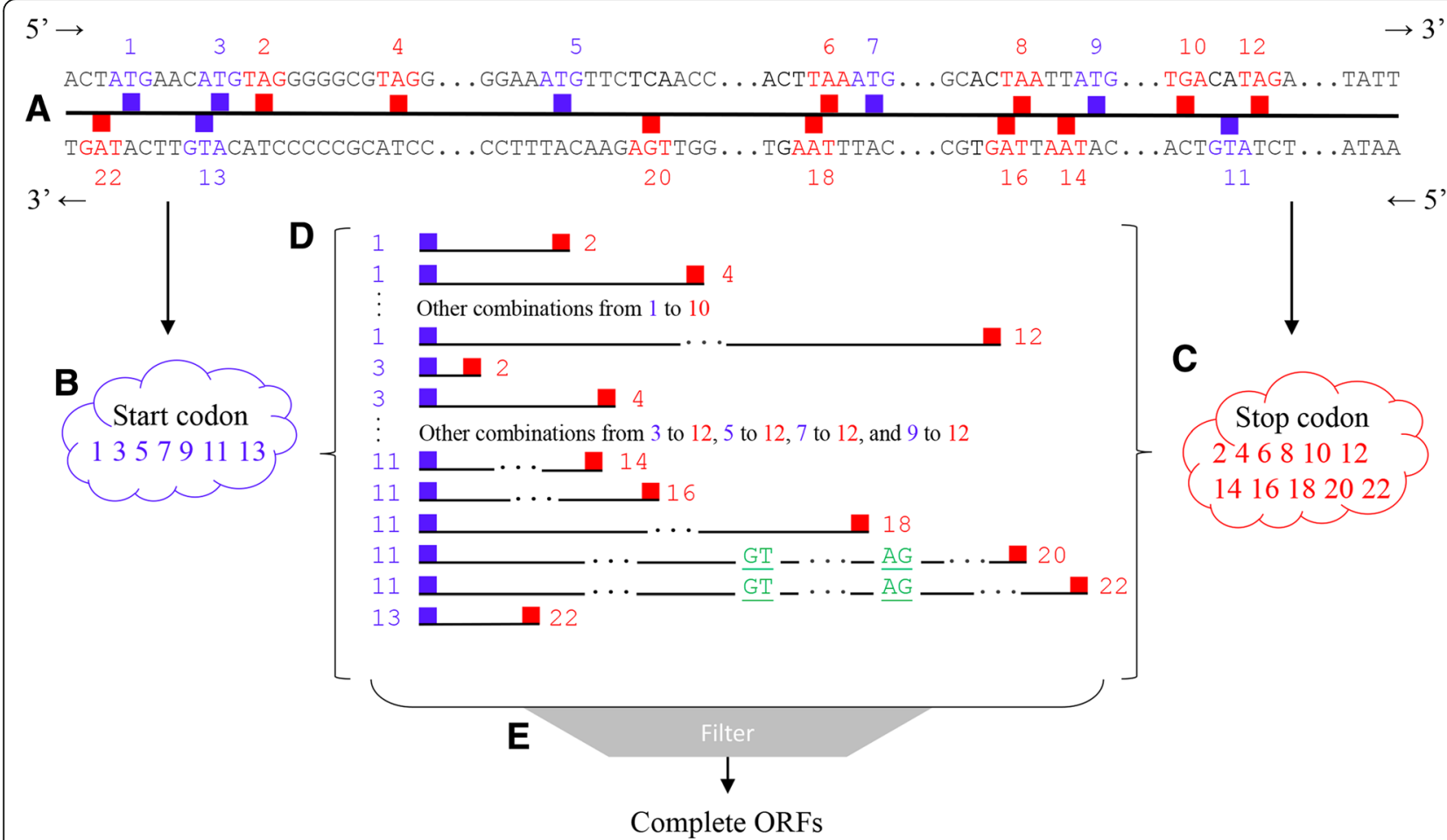

Fig. 3 Schematic representation of the VM Algorithm. Initially, the user submits a putative genomic sequence (a). Then, the algorithm scans the full-length sequence identifying all initiation codons $\left[\right.$ ATG $\left(5^{\prime} \rightarrow 3^{\prime}\right)$ and CAT $\left(3^{\prime} \rightarrow 5^{\prime}\right)$ ], which are highlighted in blue boxes and odd numbers, and stop codons $\left[T A A, T A G, T G A\left(5^{\prime} \rightarrow 3^{\prime}\right)\right.$ and TTA, CTA, TCA $\left(3^{\prime} \rightarrow 5^{\prime}\right)$, denoted in red and identified by even numbers. The initiation and stop codons are clustered separately and organized according to their numbering scheme $(\mathbf{b}, \mathbf{e}, \mathbf{c})$. Each initiation codon is tested with all stop codons to verify whether each pair can form a full-length $\operatorname{ORF}(\mathbf{d})$. All possible splicing sites GT and AG are located in the ORF (highlighted in green).

Filters are applied to evaluate the consistency of candidate ORFs and to certify that they are not truncated (e)

genes of this family, particularly Mastrevirus, which has an intron in the gene $\mathrm{C} 1: \mathrm{C} 2[49]$.

\section{Choosing the machine learning algorithm}

The Fangorn Forest method embeds two ML models built with the previously described training sets. The genus model classifies complete genomes of the nine genera in the family Geminiviridae and related satellite DNAs, using 24 attributes. The ORF model was trained to classify genes of all the above types of genomes, using 35 attributes.

In this study, three ML algorithms were tested in order to select the one that suits the classification tasks: Sequential Minimal Optimization (SMO), Random Forest (RF), and Multilayer Perceptron (MLP). Those algorithms are implemented in the suite Weka v3.8.1 [51], whose API is used in our system. The experiments performed with those methods employed the Weka API using programs in the Java programming language.

The SMO algorithm is a largely used method to solve the quadratic programming problem upon which the SVM approach is based to find the maximum-margin hyperplane for separating two classes [52]. The RF algorithm is a classification method based on decision trees, which is able to perform regression and classification. The classification of a new instance occurs by the classification of multiple trees, resulting in a consensus of those classifications through a voting procedure (ensemble) [53].

The MLP algorithm is a type of neural network that is widely used for its high predictive power in non-linear systems. Several studies report the benefits of neural networks compared to traditional statistical modeling techniques [54]. MLP features three types of artificial neuron layers: an input layer, one or more hidden (or intermediate) layers, and an output layer. Each neuron in a layer may only connect to neurons in the subsequent layer (feedforward connections). Those connections have weights (calculated in the training procedure) that define how the input data values will be processed to generate the final output. Backpropagation is the most common learning (weight adjustment) method of MLPs [54].

Those ML algorithms were run with the Weka default parameters. The generality of the resulting models was evaluated using three different techniques: (i) the use of a completely independent test set, (ii) 10 -fold cross validation, and (iii) leave-one-out (which is an $n$-fold cross validation, where $n$ is the number of instances in the training set) $[55,56]$. For each test, 
the following measures were obtained for evaluating the model performance: accuracy, precision, recall, $F$ measure, MCC (Matthews correlation coefficient) [57] (Additional file 7: Equation S1), and AUC [58]. After performing all tests, the $F$-measure (harmonic mean of precision and recall), MCC and AUC were analyzed to support our choice for the ML algorithm to be included in our system.

\section{Fangorn Forest method}

The Fangorn Forest method is composed of four fundamental parts: the family ML model, genus ML model, the VM algorithm, and the ORF ML model, as illustrated in Fig. 4. The family model classifies a complete genome as belonging to the Geminiviridae family (Fig. 4a). The genus model classifies a complete genome among eight genera of the family Geminiviridae as well as related satellite DNAs (alpha or beta satellite) (Fig. 4b). For gene prediction, the VM algorithm is first used to select candidate ORFs contained in the input genome, and, next, the ORF model classifies them within one of the classes: pre-coat, Reg, CP, AC5, REn, TrAP, Rep, Sd/p.sd, NSP, $\mathrm{MP}$, alphaRep, and betaC1. Once those classifiers are executed, their results are combined to provide an interactive visualization of the genomic organization, similarly to the structures suggested by Varsani et al. [2] Notice that the VM algorithm is not infallible, i.e., a spurious ORF might be given as input to the ORF model. F2 detects such cases by analyzing the probability distribution, across the twelve classes, yielded by the ORF model. If all probabilities are low (less than a predefined threshold - default: 0.8 ), then the putative ORF is marked as unknown (gray circle in Fig. 4f and gray

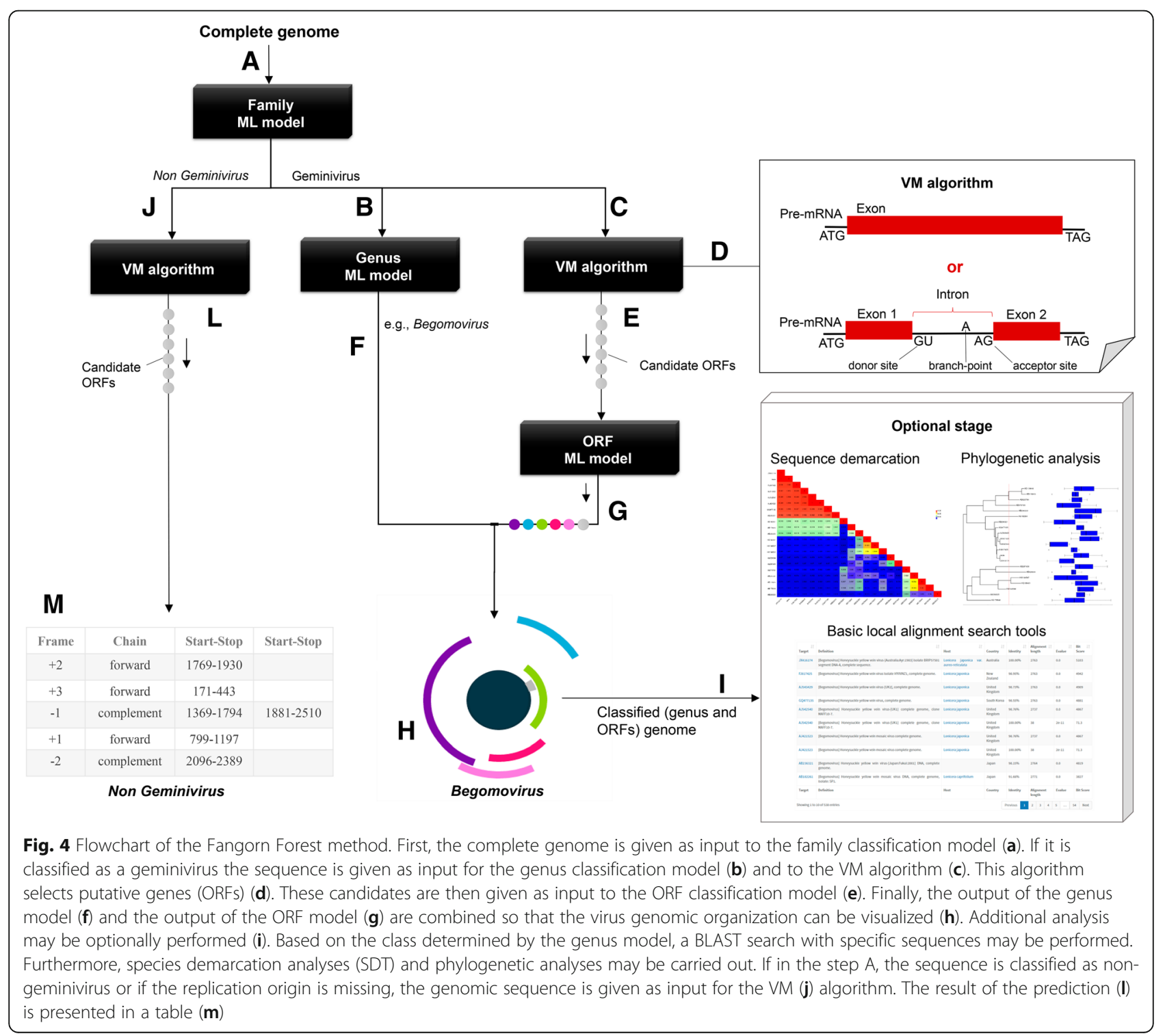


piece in Fig. 4g). DNA sequence classified as belonging to the family Geminiviridae is verified by a filter for the existence of the replication origin of geminivirus, before being fed to the second model composed of 10 classes (Fig. 4b). If the origin of replication is not found, the sequence is not submitted to the genus and gene classification model but is submitted to the VM algorithm to predict ORFs and other analysis tools (Fig. 4j). The same procedures are taken for a genomic sequence classified as a non-geminivirus sequence in the first model (Fig. 4j). If a totally unraleted genome is submitted to the method, it will be classified as non-geminivirus.

Optionally, F2 allows additional analyses using the complete genomic sequence: (i) BLASTn with e-value $1.0 \mathrm{E} 10^{-5}$, aiming to identify the closest species; (ii) phylogenetic reconstruction (BLASTn with e-value $1.0 \mathrm{E} 10^{-5}$, sequence alignment with Muscle, tree building with FastTree [59], and phytools package for tree visualization [60]); and (iii) species demarcation using the SDT software.

\section{Results and discussion}

The number of scientific studies on the family Geminiviridae has significantly increased in the last ten years (geminivirus.org:8080/geminivirusdw/statistics.jsp). The broad diversity of species, the large number of complete sequences, and the discovery of new geminiviruses have increased the complexity in determining the nomenclature and providing the taxonomic classification of geminiviruses [3, 30-32, 61-63]. Another issue in the family Geminiviridae concerns some particular genes in some species of the genus Mastrevirus, post-transcriptional changes may occur in primary gene transcripts, such as for MSV, whose genome holds gene C1:C2 [49]. Posttranscriptional processing of genes is common in eukaryotes and rare in prokaryotes. It occurs through a series of reactions catalyzed by the host spliceosome or selfsplicing mechanisms [64]. The traditional tools to predict ORFs, such as ORF Finder, have not been adapted for the possibility of splicing. Other consolidated tools, such as AUGUSTUS, Geneid (both adapted for Eukaryote) and Prodigal (adapted for Prokaryotes), are still limited to identify all ORFs encoded by a given genome sequence of geminivirus species. These tools consider common features for organisms that have larger genomes with more complex promoters.

To mitigate all these issues, the present study developed the family and genus classification model along with the VM algorithm, for ORF extraction, associated with an ORF classification model so that a geminivirus genome sequence could be classified into one of genera in the Geminiviridae family, and the genes in this sequence could be easily identified. The results to validate our method are presented below. Notice that we do not provide here a comparison between methods, as, to our knowledge, there is no known approach, with similar intent, proposed specifically to geminiviruses, and that works in an ab initio manner (i.e., only the input sequence itself is analyzed). Thus, no homology analysis procedure, which is the usual approach in general, is used in our case.

\section{Attribute analysis results}

Additional file 3: Tables S2, Additional file 4: Table S3 and Additional file 5: Table S4 show the results of the attribute analysis using IG and RELIEFF. Both methods agreed on the relevance of some top and low-ranked attributes, although the evaluation of many others attributes presented highly dissimilar rank positions comparing the outputs of those algorithms. Most importantly, none of the attributes presented null relevance in both ranks. In fact, we tried to remove some low-ranked attributes for all processes, family, genus and ORF model training. It turns out that all attempts to eliminate any of the attributes caused a decrease in performance of the resultant models

The relevance of all proposed attributes for building both models was corroborated by histograms, density plots and boxplots. An example is provided in Fig. 5 for the attribute 'length' used in ORF classification. The histogram and density plot demonstrate diverse distributions of that attribute across the classes. Additionally, the boxplot shows very distinct means and standard deviations of the same attribute when the classes are compared. Additional file 6 shows these plots for all attributes in both training sets (genus and ORF). The same conclusions about the distribution diversity across the classes can be drawn for the other attributes in both classification tasks. Based on these analyses, we decided to keep all proposed attributes in the training sets used to construct the F2 models.

\section{Performance of the ML models}

Tables 2, 3 and 4 show the performance of the models for family, genus and ORF classification, which were built with MLP, SMO, and RF, using the default parameters of Weka (see Additional file 8: Table S5 for more details). It can be seen that MLP and RF are superior than SMO for genus classification. For ORF classification, on the other hand, all methods performed well. Inspecting the $F$-measure, it is difficult to choose between MLP and RF. MLP was slightly better for genus classification, while RF presented slightly superior values for ORF classification. However, based on the results shown in Tables 2, 3 and 4, we chose RF as the classifier for both genus and ORF for two reasons: (i) RF presented the greatest AUC value in all tests for both classification tasks, which means more 


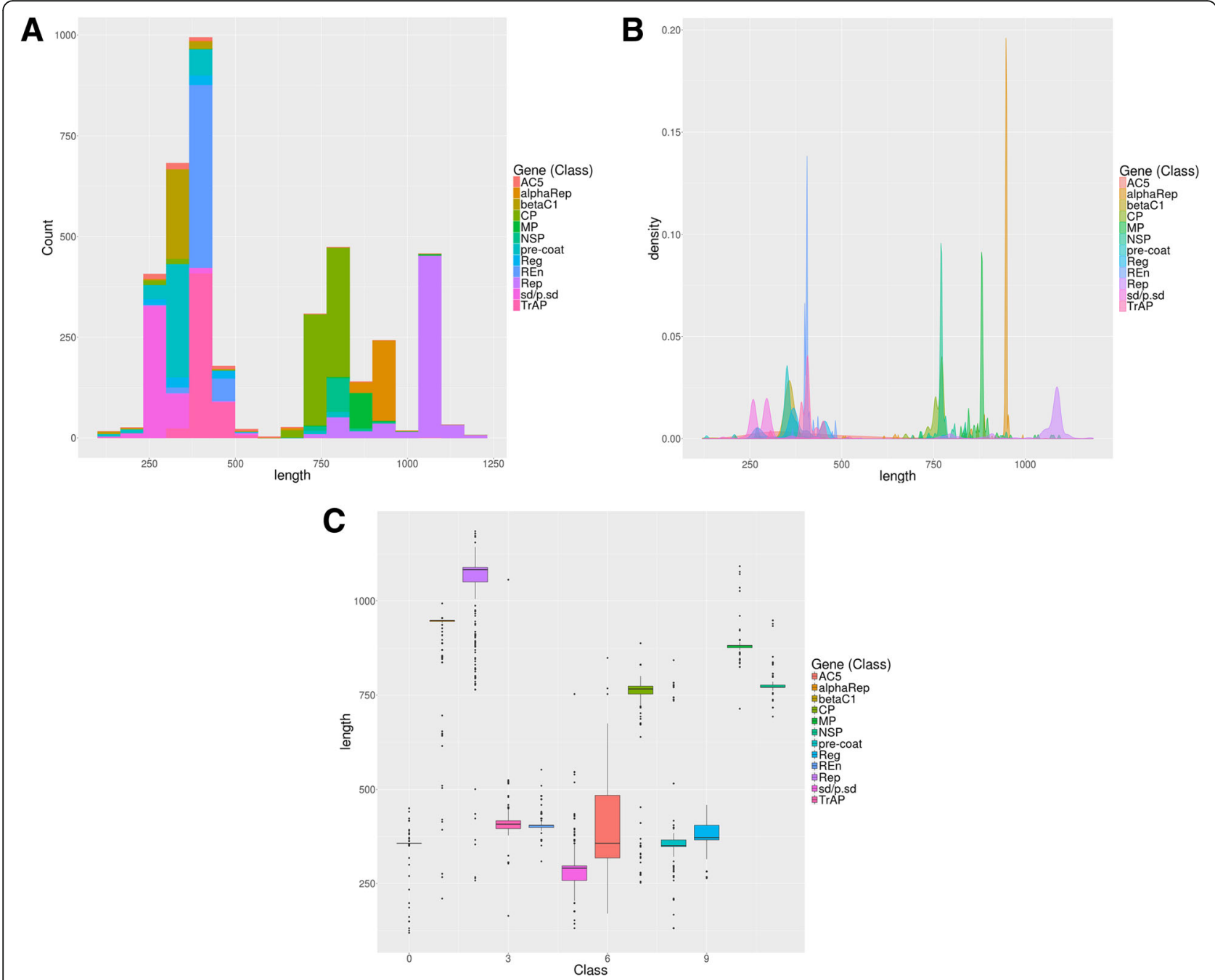

Fig. 5 Exploratory analysis of the sequence length attribute. a) Histogram. b Density plot. c Boxplot

Table 2 Performance of the family classification model using default parameters of Weka

\begin{tabular}{|c|c|c|c|c|c|c|c|}
\hline \multirow[t]{2}{*}{ Type of evaluation } & \multirow[t]{2}{*}{ ML algorithm } & \multicolumn{6}{|c|}{ Weighted average among all classes } \\
\hline & & Accuracy & Precision & Recall & F-Measure & MCC & AUC \\
\hline \multirow[t]{3}{*}{ Using a test set } & MLP & 0.9444 & 0.946 & 0.944 & 0.944 & 0.891 & 0.969 \\
\hline & $\mathrm{SMO}$ & 0.8107 & 0.815 & 0.811 & 0.810 & 0.625 & 0.810 \\
\hline & RF & 0.9542 & 0.955 & 0.954 & 0.954 & 0.909 & 0.988 \\
\hline \multirow[t]{3}{*}{ 10-fold cross validation } & MLP & 0.9369 & 0.937 & 0.937 & 0.937 & 0.871 & 0.972 \\
\hline & $\mathrm{SMO}$ & 0.8568 & 0.861 & 0.857 & 0.855 & 0.709 & 0.844 \\
\hline & $\mathrm{RF}$ & 0.9601 & 0.960 & 0.960 & 0.960 & 0.919 & 0.992 \\
\hline \multirow[t]{3}{*}{ Leave-one-out } & MLP & 0.944 & 0.944 & 0.944 & 0.944 & 0.886 & 0.975 \\
\hline & $\mathrm{SMO}$ & 85.597 & 0.860 & 0.856 & 0.854 & 0.707 & 0.843 \\
\hline & RF & 96.228 & 0.963 & 0.962 & 0.962 & 0.923 & 0.992 \\
\hline \multirow[t]{3}{*}{ Mean performance } & MLP & 0.9420 & 0.9430 & 0.9417 & 0.9417 & 0.8843 & 0.9700 \\
\hline & $\mathrm{SMO}$ & 0.8411 & 0.8433 & 0.8413 & 0.8339 & 0.6803 & 0.8323 \\
\hline & RF & 0.9588 & 0.9533 & 0.9586 & 0.9586 & 0.917 & 0.9906 \\
\hline
\end{tabular}


Table 3 Performance of the genus classification model using default parameters of Weka

\begin{tabular}{|c|c|c|c|c|c|c|c|}
\hline \multirow[t]{2}{*}{ Type of evaluation } & \multirow{2}{*}{$\begin{array}{l}\text { ML } \\
\text { algorithm }\end{array}$} & \multicolumn{6}{|c|}{ Weighted average among all classes } \\
\hline & & Accuracy & Precision & Recall & F-Measure & MCC & AUC \\
\hline \multirow[t]{3}{*}{ Using a test set } & MLP & 0.941 & 0.963 & 0.941 & 0.951 & 0.8940 & 0.971 \\
\hline & SMO & 0.835 & 0.865 & 0.835 & 0.795 & 0.6340 & 0.816 \\
\hline & RF & 0.934 & 0.941 & 0.934 & 0.936 & 0.8750 & 0.988 \\
\hline \multirow[t]{3}{*}{ 10-fold cross validation } & MLP & 0.970 & 0.970 & 0.971 & 0.970 & 0.9610 & 0.991 \\
\hline & SMO & 0.920 & 0.901 & 0.920 & 0.906 & 0.8850 & 0.962 \\
\hline & RF & 0.966 & 0.966 & 0.966 & 0.965 & 0.9510 & 0.997 \\
\hline \multirow[t]{3}{*}{ Leave-one-out } & MLP & 0,971 & 0,971 & 0,972 & 0,960 & 0.9920 & 0.995 \\
\hline & SMO & 0.944 & 0.938 & 0.945 & 0.939 & 0.8810 & 0.946 \\
\hline & RF & 0.991 & 0.991 & 0.991 & 0.991 & 0.9550 & 0.999 \\
\hline \multirow[t]{3}{*}{ Mean performance } & MLP & 0.966 & 0.974 & 0.967 & 0.970 & 0.9490 & 0.986 \\
\hline & SMO & 0.900 & 0.901 & 0.900 & 0.880 & 0.8800 & 0.908 \\
\hline & $\mathrm{RF}$ & 0.964 & 0.966 & 0.964 & 0.964 & 0.9238 & 0.995 \\
\hline
\end{tabular}

coherent output probabilities; and (ii) the training time for RF is much shorter compared with the other methods.

Most importantly, RF demonstrated a very high prediction power. For the classification model of the family Geminividae, the RF algorithm achieves mean performance of $0.9588 .0 .9533,0.9586,0.9586,0.917,0.9906$ of accuracy, precision, recall, F-measure, MCC and AUC, respectively. The mean performance of RF for genus classification was $0.964,0.966 .0 .964,0.964,0.923,0.995$ of accuracy, precision, recall, F-measure, MCC and AUC, respectively. For ORF classification, RF achieved the mean values $0.977,0.978,0.978,0.978,0.975,0.997$ of accuracy, precision, recall, F-measure, MCC and AUC, respectively.

To evaluate the overall pipeline, a set of sequences of plant viruses, sequences from the Circoviridae
Family (circular single-stranded DNA animal virus) and artificially generated sequences were submitted manually to the web interface of the pipeline method. The method was adjusted with the threshold of 0.5 (default) for family and ORF classifications. In this test, F2 achieved accuracy, precision, recall and FMeasure of $0.9343,0.9343,0.9343$, and 0.9343 , respectively, for the correct identification of genomes of non-geminiviruses or geminiviruses and their genus (Additional file 9). In addition, a partial begomovirus sequence (EF591125-begomovirus), which does not encode a protein, was not classified as geminivirus. Likewise, a defective KT099181 sequence of betasatellites was not cataloged as a geminivirus-related DNA satellite. These examples demonstrated that defective begomovirus genomes, which did not display the

Table 4 Performance of the gene classification model using default parameters of Weka

\begin{tabular}{|c|c|c|c|c|c|c|c|}
\hline \multirow[t]{2}{*}{ Type of evaluation } & \multirow[t]{2}{*}{ ML algorithm } & \multicolumn{6}{|c|}{ Weighted average among all classes } \\
\hline & & Accuracy & Precision & Recall & F-Measure & MCC & $A \cup C$ \\
\hline \multirow[t]{3}{*}{ Using a test set } & MLP & 0.972 & 0.973 & 0.973 & 0.972 & 0.968 & 0.985 \\
\hline & SMO & 0.976 & 0.977 & 0.976 & 0.976 & 0.973 & 0.995 \\
\hline & RF & 0.981 & 0.982 & 0.982 & 0.982 & 0.979 & 0.998 \\
\hline \multirow[t]{3}{*}{ 10-fold cross validation } & MLP & 0.970 & 0.971 & 0.971 & 0.971 & 0.967 & 0.994 \\
\hline & SMO & 0.972 & 0.973 & 0.973 & 0.973 & 0.969 & 0.994 \\
\hline & RF & 0.976 & 0.977 & 0.977 & 0.977 & 0.974 & 0.997 \\
\hline \multirow[t]{3}{*}{ Leave-one-out } & MLP & 0.970 & 0.970 & 0.970 & 0.970 & 0.966 & 0.994 \\
\hline & SMO & 0.9727 & 0.973 & 0.973 & 0.973 & 0.969 & 0.994 \\
\hline & RF & 0.9759 & 0.976 & 0.976 & 0.976 & 0.973 & 0.997 \\
\hline \multirow[t]{3}{*}{ Mean performance } & MLP & 0.9707 & 0.9713 & 0.9713 & 0.9710 & 0.9670 & 0.9910 \\
\hline & SMO & 0.9736 & 0.9743 & 0.9740 & 0.9740 & 0.9703 & 0.9943 \\
\hline & RF & 0.9776 & 0.9783 & 0.9783 & 0.9783 & 0.9753 & 0.9973 \\
\hline
\end{tabular}


genomic structure of geminivirus were not recognized as geminiviruses.

Some geminivirus genomes exhibit considerable similarity with the genomic structure of different families of ssDNA viruses (i.e. circoviruses and parvoviruses) (Additional file 9). Thus, genomic sequences of Family Circovidae and Parvoviridae were confronted to F2 and three of 20 sequences were classified as geminiviruses with low probability. Furthermore, the predicted ORFs were not classified as geminivirus ORFs within the established limit as default. Random sequences with geminivirus origin of replication were created and compared against the F2 method. Neither of these sequences were classified as geminiviruses nor the predicted ORFs were classified as geminiviral ORFs.

In addition to predicting family and gender, the F2 method can predict ORFs and classify sequences of geminivirus-specific ORFs (genes). Some species encode two to seven genes only in the component A. Most sequences are short and important to complete the infectious cycle of the virus. Like the ORF finder, some other tools can identify ORFs; however, they did not identify introns and hence they fail to annotate some genes. The AUGUSTUS tool is widely consolidated and widely used in genome projects to perform a prediction of eukaryotic genes. We confronted AUGUSTUS and ORF finder by performing a gene prediction for the most common begomovirus sequences (AF416742, AF448058, AF241479, AF126406, DQ026296). For each of these sequences, the AUGUSTUS algorithm only identified two ORFs, whereas these genomes encode six to seven genes. Mastervirus sequences (KY618115, KF806701, KJ187748, KC172663, HQ113104) were also used, however, few genes and no introns were identified. The ORF finder identified almost all geminivirus genes, except the ones with introns. The methodology proposed by the F2 method can complement these tools, as it is efficient to annotate all geminivirus genes.

\section{Conclusion}

Geminiviridae is an important plant virus family, as it represents a serious threat to agriculture and food security. Identifying genera of this family requires caution and has become a challenge due to a large number of sequences available in databases. Moreover, advanced knowledge in taxonomy and bioinformatics analyses is currently required.

As a result of this research, a new method based on machine learning techniques, called Fangorn Forest, is proposed to automatize the identification of genera and genes of the family Geminiviridae. This method is composed of four fundamental parts. The family and genus classification module is able to classify a complete genome within one of eight genera of the family Geminiviridae or associated satellite DNAs (alpha or beta satellite). Another important component is the algorithm for ORF identification, called here Viaduc de Millau (VM), created for the specific peculiarities of the family Geminiviridae, which are not covered by other general-purpose ORF predictors, such as ORF finder. VM is used in conjunction with the third important part of our system. This is the ORF classification procedure that classifies the ORFs extracted by VM according to the typical gene types encountered in geminiviruses. Both classifiers, for genus and ORF, are highly accurate, as could be seen in the presented results.

It is also worth mentioning the additional stages that can be performed with the input sequence. Our system may optionally use the SDT tool for species-demarcation, and perform phylogenetic analyses, which greatly facilitate the study under consideration. To this purpose, F2 is adapted to act autonomously based on the genus classification, whose result redirects the analysis to specific databases for the identified genus, so that an appropriate set of sequences can be used to perform the analyses.

We stress the importance of automatizing genus and ORF classification, with high accuracy, with a special focus on geminiviruses, resulting in a powerful customized system for this type of virus that causes expressive economic impacts. The method is freely accessible at http://geminivirus.org:8080/geminivirusdw/discoveryGeminivirus.jsp.

\section{Additional files}

Additional file 1: Table S1. Number of instances (sequences) of each family, genus, and respective ORFs, contained in the datasets. (DOC 78 kb)

Additional file 2: This file presents the accession numbers of the complete genomic sequences that make up the training and test sets. (XLS $1650 \mathrm{~kb}$ )

Additional file 3: Table S2. The IG, RELIEFF ranks of attributes in the family training set. Attributes are sorted by the IG rank. (DOC $53 \mathrm{~kb}$ )

Additional file 4: Table S3. The IG, RELIEFF ranks of attributes in the genus training set. Attributes are sorted by the IG rank. (DOC 52 kb)

Additional file 5: Table S4. The IG, RELIEFF ranks of attributes in the ORF training set. Attributes are sorted by the IG rank. (DOC 62 kb)

Additional file 6: This file shows plots (histogram, density and boxplot) related to the attributes of the ORF and genus training sets. (PDF $8536 \mathrm{~kb}$ )

Additional file 7: Equations S1. Model assessment measures. (DOCX $16 \mathrm{~kb}$ )

Additional file 8: Table S5. Performance results of the tests (performed with the Weka) for the models of family, genus and gene classification. (DOC $147 \mathrm{~kb}$ )

Additional file 9: This file presents the pipeline evaluation with geminiviruses sequences, plant viruses, circoviruses and fake sequences. (XLS $44 \mathrm{~kb}$ )

\section{Abbreviations}

BGMD: Bean golden mosaic disease; CDS: Coding DNA sequence;

CLCuD: Cotton leaf curl disease; CMD: Cassava mosaic disease; F2: Fangorn forest; IG: Information gain; ML: Machine learning; MLP: Multilayer perceptron; MP: Movement protein; MSD: Maize streak disease; MSV: Maize 
streak virus; NSP: Nuclear transport protein; ORFs: Open reading frames; RCA: Rolling circle amplification; RF: Random forest; SDT: Species demarcation analyses; SMO: Sequential minimal optimization; SVM: Support vector machine; TYLCD: Tomato yellow leaf curl virus; UTR: Untranslated region; VM: Viaduc de Millau; WDVD: Wheat dwarf virus disease

\section{Acknowledgements}

The authors are thankful to the Universidade Federal de Viçosa (UFV) National Institute of Science and Technology in Plant-Pest Interactions (INCTIPP), Computer Science Department - UFV and Division of Support to Scientific and Technological Development - UFV.

\section{Funding}

This research was financially supported by the following grants from Brazilian government agencies: Conselho Nacional de Desenvolvimento Científico e Tecnológico (CNPq), Fundação de Amparo à Pesquisa do Estado de Minas Gerais (FAPEMIG), Coordenação de Aperfeiçoamento de Pessoal de Nível Superior (CAPES) and National Institute of Science and Technology in Plant-Pest Interactions (INCTIPP). The funding bodies did not play any role in the design of the study, in the analysis and interpretation of data.

\section{Availability of data and materials}

The datasets generated during the current study are available in the geminivirus data warehouse repository, geminivirus.org.

\section{Author's contributions}

JCFS suggested this study and designed this geminivirus.org. JCFS and TFMC implemented the software and provided the in silico validation of the method. All authors helped to draft the manuscript. FRC and EPBF supervised this study. All authors read and approved the final manuscript.

\section{Ethics approval and consent to participate}

Not applicable.

\section{Consent for publication}

Not applicable.

\section{Competing interests}

The authors declare that they have no competing interests.

\section{Author details}

'Department of Informatics, Universidade Federal de Viçosa, Viçosa, Minas Gerais 36570-900, Brazil. ${ }^{2}$ National Institute of Science and Technology in Plant-Pest Interactions/BIOAGRO, Campus Universitário, Viçosa, Minas Gerais 36570-900, Brazil. ${ }^{3}$ Department of Biochemistry and Molecular Biology, Universidade Federal de Viçosa, Campus Universitário, Viçosa, Minas Gerais 36570-900, Brazil. ${ }^{4}$ Department of Production Engineering, Universidade Federal Fluminense, Rua Domingos Silvério, s/n, Bairro Quitandinha, Petrópolis, Rio de Janeiro 25650-050, Brazil.

\section{Received: 1 May 2017 Accepted: 20 September 2017}

Published online: 30 September 2017

\section{References}

1. Hanley-Bowdoin L, Bejarano ER, Robertson D, Mansoor S. Geminiviruses: masters at redirecting and reprogramming plant processes. Nat Rev Microbiol. 2013;11(11):777-88.

2. Varsani A, Navas-Castillo J, Moriones E, Hernández-Zepeda C, Idris A, Brown $\mathrm{JK}$, Zerbini FM, Martin DP. Establishment of three new genera in the family Geminiviridae: Becurtovirus, Eragrovirus and Turncurtovirus. Arch Virol. 2014; 159(8):2193-203.

3. Roumagnac P, Granier M, Bernardo P, Deshoux M, Ferdinand R, Galzi S, Fernandez E, Julian C, et al. Alfalfa leaf curl virus: An aphid-transmitted geminivirus. J Virol. 2015;89(18):9683-8.

4. Varsani A, Roumagnac P, Fuchs M, Navas-Castillo J, Moriones E, Idris A, Martin DP. Capulavirus and Grablovirus: two new genera in the family Geminiviridae. Arch Virol. 2017;162 1819-1831-13

5. Davies JW, Stanley J, Donson J, Mullineaux PM, Boulton MI. Structure and replication of geminivirus genomes. J Cell Sci. 1987;7:95-107.

6. Harrison BD. Advances in geminivirus research. Annu Rev Phytopathol. 1985; 23(1):55-82
7. Howarth AJ, Vandemark GJ. Phylogeny of geminiviruses. J Gen Virol. 1989; 70(10):2717-27.

8. Rybicki EP. A phylogenetic and evolutionary justification for three genera of Geminiviridae. Arch Virol. 1994;139(1-2):49-77.

9. Briddon RW, Patil BL, Bagewadi B, Nawaz-ul-Rehman MS, Fauquet CM. Distinct evolutionary histories of the DNA-A and DNA-B components of bipartite begomoviruses. BMC Evol Biol. 2010;10(1):1.

10. Melgarejo TA, Kon T, Rojas MR, Paz-Carrasco L, Zerbini FM, Gilbertson RL. Characterization of a New World Monopartite Begomovirus Causing Leaf Curl Disease of Tomato in Ecuador and Peru Reveals a New Direction in Geminivirus Evolution. J Virol. 2013:87(10):5397-413.

11. Parrella G, Scassillo L, Crescenzi A, Nappo A. Typing of tomato yellow leaf curl viruses and their vector in Italy. Commun Agric Appl Biol Sci. 2005;71(3):229-1236.

12. De Bruyn A, Harimalala M, Hoareau M, Ranomenjanahary S, Reynaud B, Lefeuvre P, Lett JM. Asystasia mosaic madagascar virus: a novel bipartite begomovirus infecting the weed Asystasia gangetica in Madagascar. Arch Virol. 2015;160(6):1589-91.

13. Nagendran K, Satya V, Mohankumar S, Karthikeyan G. Molecular characterization of a distinct bipartite Begomovirus species infecting ivy gourd (Coccinia grandis I.) in Tamil Nadu, India. Virus Genes.2016;52(1):1-6.

14. Nannini M, Foddi F, Murgia G, Pisci R, Sanna F, Testa M, Accotto G. An epidemiological survey of TYLCD in Southern Sardinia (Italy). Commun Agric Appl Biol Sci. 2008;74(3):831-41.

15. Manurung B, Witsack W, Mehner S, Grüntzig M, Fuchs E. The epidemiology of Wheat dwarf virus in relation to occurrence of the leafhopper Psammotettix alienus in Middle-Germany. Virus Res. 2004;100(1):109-13.

16. Schubert J, Habekuß A, Wu B, Thieme T, Wang X. Analysis of complete genomes of isolates of the Wheat dwarf virus from new geographical locations and descriptions of their defective forms. Virus Genes. 2014;48(1):133-9.

17. Patil BL, Fauquet CM. Cassava mosaic geminiviruses: actual knowledge and perspectives. Mol Plant Pathol. 2009;10(5):685-701.

18. Shepherd DN, Martin DP, van der Walt E, Dent K, Varsani A, Rybicki EP: Maize streak virus: an old and complex 'emerging' pathogen. Mol Plant Pathol 2010;11(1):1-12

19. Briddon RW, Markham P. Cotton leaf curl virus disease. Virus Res. 2000; 71(1):151-9.

20. Sattar MN, Kvarnheden A, Saeed M, Briddon RW. Cotton leaf curl disease-an emerging threat to cotton production worldwide. J Gen Virol. 2013;94(4): 695-710.

21. Faria JC, Maxwell DP. Variability in Geminivirus Isolates Associated with Phaseolus spp. in Brazil. Phytopathology. 1999;89(3):262-8.

22. Li F, Xu X, Huang C, Gu Z, Cao L, Hu T, Ding M, Li Z, Zhou X. The AC5 protein encoded by Mungbean yellow mosaic India virus is a pathogenicity determinant that suppresses RNA silencing-based antiviral defenses. New Phytol. 2015;208(2):555-69.

23. Krenz $B$, Jeske $H$, Kleinow $T$. The induction of stromule formation by a plant DNA-virus in epidermal leaf tissues suggests a novel intra-and intercellular macromolecular trafficking route. Front Plant Sci. 2012;3:291.

24. Zhou X. Advances in understanding begomovirus satellites. Annu Rev Phytopathol. 2013;51:357-81

25. Briddon RW, Bull SE, Amin I, Idris AM, Mansoor S, Bedford ID, Dhawan P, Rishi N, Siwatch SS, Abdel-Salam AM, Brown JK. Diversity of DNA $\beta$, a satellite molecule associated with some monopartite begomoviruses. Virology. 2003;312(1):106-21.

26. Kumar J, Kumar J, Singh SP, Tuli R. $\beta C 1$ is a pathogenicity determinant: not only for begomoviruses but also for a mastrevirus. Arch Virol. 2014;159(11):3071-6.

27. Briddon RW, Stanley J. Subviral agents associated with plant single-stranded DNA viruses. Virology. 2006;344(1):198-210.

28. Briddon RW, Bull SE, Amin I, Mansoor S, Bedford ID, Rishi N, Siwatch SS, Zafar Y, Abdel-Salam AM, Markham PG. Diversity of DNA 1: a satellite-like molecule associated with monopartite begomovirus-DNA $\beta$ complexes. Virology. 2004;324(2):462-74

29. Paprotka $T$, Metzler $V$, Jeske $H$. The first DNA 1-like a satellites in association with New World begomoviruses in natural infections. Virology. 2010;404(2):148-57.

30. Muhire B, Martin DP, Brown JK, Navas-Castillo J, Moriones E, Zerbini FM Rivera-Bustamante R, Malathi V, Briddon RW, Varsani A. A genome-wide pairwise-identity-based proposal for the classification of viruses in the genus Mastrevirus (family Geminiviridae). Arch Virol. 2013;158(6):1411-24.

31. Brown JK, Zerbini FM, Navas-Castillo J, Moriones E, Ramos-Sobrinho R, Silva JC, Fiallo-Olivé E, Briddon RW, Hernández-Zepeda C, Idris A, et al. Revision of 
Begomovirus taxonomy based on pairwise sequence comparisons. Arch Virol. 2015;160(6):1593-619.

32. Briddon R, Brown J, Moriones E, Stanley J, Zerbini FM, Zhou X, Fauquet C. Recommendations for the classification and nomenclature of the dna- $\beta$ satellites of begomoviruses. Arch Virol. 2008;153(4):763-81.

33. Inoue-Nagata AK, Albuquerque LC, Rocha WB, Nagata T. A simple method for cloning the complete begomovirus genome using the bacteriophage ب29 dna polymerase. J Virol Methods. 2004;116(2):209-11.

34. Edgar RC. MUSCLE: multiple sequence alignment with high accuracy and high throughput. Nucleic Acids Res. 2004;32(5):1792-7.

35. Katoh K, Standley DM. MAFFT multiple sequence alignment software version 7: improvements in performance and usability. Mol Biol Evol. 2013;30(4):772-80

36. Li K-B. ClustalW-MPI: ClustalW analysis using distributed and parallel computing. Bioinformatics. 2003;19(12):1585-6.

37. Altschul SF, Madden TL, Schäffer AA, Zhang J, Zhang Z, Miller W, Lipman DJ. Gapped BLAST and PSI-BLAST: a new generation of protein database search programs. Nucleic Acids Res. 1997;25(17):3389-402.

38. Rocha CS, Castillo-Urquiza GP, Lima AT, Silva FN, Xavier CA, Hora-Júnior BT, Beserra-Júnior JE, Malta AW, Martin DP, Varsani A, et al. Brazilian begomovirus populations are highly recombinant, rapidly evolving, and segregated based on geographical location. J Virol. 2013;87(10):5784-99.

39. Muhire BM, Varsani A, Martin DP. SDT: A Virus Classification Tool Based on Pairwise Sequence Alignment and Identity Calculation. PLoS One. 2014;9(9):108277.

40. Varsani A, Martin DP, Navas-Castillo J, Moriones E, Hernández-Zepeda C, Idris A, Zerbini FM, Brown JK. Revisiting the classification of curtoviruses based on genome-wide pairwise identity. Arch Virol. 2014;159(7):1873-82.

41. Silva JCF, Carvalho TFM, Basso MF, Deguchi M, Pereira WA. et al. Geminivirus Data Warehouse: A database enriched with machine learning approaches. BMC Bioinformatics. 2016 (in press).

42. Briddon RW, Bedford ID, Tsai JH, Markham PG. Analysis of the Nucleotide Sequence of the Treehopper-Transmitted Geminivirus, Tomato Pseudo-Curly Top Virus, Suggests a Recombinant Origin. Virology. 1996;219(2):387-94.

43. Arguello-Astorga G, Lopez-Ochoa L, Kong LJ, Orozco BM, Settlage SB, Hanley-Bowdoin L. A Novel Motif in Geminivirus Replication Proteins Interacts with the Plant Retinoblastoma-Related Protein. J Virol. 2004; 78(9):4817-26.

44. Yang Y, Pedersen JO. A comparative study on feature selection in text categorization. In: ICML 1997:97:412-420.

45. KIRA K, RENDELL LA. A practical approach to feature selection. In: Proceedings of the ninth international workshop on Machine learning. California: Morgan Kaufmann; 1992. p. 249-56.

46. Shi Y, Guo Y, Hu Y, Li M. Position-specific prediction of methylation sites from sequence conservation based on information theory. Sci Rep. 2015:5:12403.

47. Wen PP, Shi SP, Xu HD, Wang LN, Qiu JD. Accurate in silico prediction of species-specific methylation sites based on information gain feature optimization. Bioinformatics. 2016;32(20):3107-15.

48. Kononenko I. Estimating attributes: analysis and extensions of RELIEF. European conference on machine learning 1994;784:171-182.

49. Wright E, Heckel T, Groenendijk J, Davies J, Boulton M. Splicing features in maize streak virus virion-and complementary-sense gene expression. Plant J. 1997;12(6):1285-97.

50. Nelson DL, Lehninger AL, Cox MM. Lehninger Principles of Biochemistry. 5th ed. New York: 2008.

51. Hall M, Frank E, Holmes G, Pfahringer B, Reutemann P, Witten IH. The WEKA data mining software: an update. SIGKDD Explor. 2009;11(1):10-8.

52. Platt JC. 12 Fast Training of Support Vector Machines using Sequential Minimal Optimization. Advances in kernel methods 1999;1:185-208.

53. Breiman L. Random Forests. Mach Learn. 2001:45(1):5-32.

54. Gardner MW, Dorling S. Artificial neural networks (the multilayer perceptron) - a review of applications in the atmospheric sciences. Atmos Environ. 1998;32(14):2627-36.

55. Kohavi R, et al.: A study of cross-validation and bootstrap for accuracy estimation and model selection. In: ljcai 1995;14:1137-1145.

56. Arlot $\mathrm{S}$, Celisse A. A survey of cross-validation procedures for model selection. Stat Surv. 2010;4:40-79.

57. Matthews BW. Comparison of the predicted and observed secondary structure of T4 phage lysozyme. Biochim Biophys Acta. 1975;405(2):442-51.
58. Bradley AP. The use of the area under the ROC curve in the evaluation of machine learning algorithms. Pattern Recogn. 1997;30(7):1145-59.

59. Price MN, Dehal PS, Arkin AP. FastTree 2-approximately maximumlikelihood trees for large alignments. PLoS One. 2010;5(3):9490.

60. Revell $\sqcup$. Phytools: an R package for phylogenetic comparative biology (and other things). Methods Ecol Evol. 2012;3(2):217-23.

61. Lim S, Igori D, Zhao F, Moon JS, Cho IS, Choi GS. First report of Grapevine red blotch-associated virus on Grapevine in Korea. Plant Dis. 2016;100(9):1957.

62. Ng TFF, Marine R, Wang C, Simmonds P, Kapusinszky B, Bodhidatta L, Oderinde BS, Wommack KE, Delwart E. High Variety of Known and New RNA and DNA Viruses of Diverse Origins in Untreated Sewage. J Virol. 2012; 86(22):12161-75.

63. Perry KL, McLane H, Hyder MZ, Dangl GS, Thompson JR, Fuchs MF. Grapevine red blotch-associated virus is Present in Free-Living Vitis spp. Proximal to Cultivated Grapevines. Phytopathol. 2016;106(6):663-70.

64. Clancy S. RNA splicing introns, exons and spliceosome. Nat Educ. 2008;1(1):31.

\section{Submit your next manuscript to BioMed Central and we will help you at every step:}

- We accept pre-submission inquiries

- Our selector tool helps you to find the most relevant journal

- We provide round the clock customer support

- Convenient online submission

- Thorough peer review

- Inclusion in PubMed and all major indexing services

- Maximum visibility for your research

Submit your manuscript at www.biomedcentral.com/submit 